\title{
EXPONENTIAL GROWTH AND THE SPECTRUM OF THE LAPLACIAN
}

\author{
ROBERT BROOKS ${ }^{1}$
}

\begin{abstract}
ABSTRACr. Conditions are given on a noncompact manifold which allow one to conclude that $\mathbf{O}$ is in the spectrum of the Laplacian on $M$.
\end{abstract}

Let $M$ be a smooth, complete, noncompact Riemannian manifold, and denote by $\Delta$ the Laplace-Beltrami operator of $M$ acting on $L^{2}(M)$.

$M$ is said to have exponential growth if, for some point $y_{0} \in M$, the volume $V\left(r, y_{0}\right)$ of the ball $B\left(r, y_{0}\right)$ of radius $r$ about $y_{0}$ satisfies the estimate

$$
V\left(r, y_{0}\right) \leqslant C \cdot e^{\mu r}
$$

for some positive constants $C$ and $\mu$ independent of $r$. An easy estimate shows that if this estimate holds for some $y_{0}$, then it holds for all $y_{0}$ and the same $\mu$, although with perhaps different $C$.

The exponential growth of $M$ is then the infimum of all $\mu$ giving such an inequality, and is equal to $\overline{\lim }_{r \rightarrow \infty} r^{-1} \log \left(V\left(r, y_{0}\right)\right)$.

$M$ is said to have subexponential growth if the exponential growth of $M$ is $\mathbf{0 .}$

We denote by $H_{t}(x, y)$ the heat kernel on $M$. By definition, it is the fundamental solution to the heat equation, that is,

(i) for $y_{0}$ fixed, we have

$$
(\Delta+\partial / \partial t)\left[H_{t}\left(x, y_{0}\right)\right]=0 \text { for } t>0
$$

(ii) as $t \rightarrow 0, H_{t}(x, y)$ converges to the Dirac $\delta$-distribution.

The existence and uniqueness of $H_{t}(x, y)$ are established in [2]. See also [4] for many properties of $H_{t}(x, y)$ used below.

The object of this note is to prove:

THEOREM. Assume that $M$ satisfies the following three conditions:

(a) There is a constant a such that the sectional curvatures of $M$ are $>-a$.

(b) There is a constant $C$ such that

$$
H_{1}(x, y) \leqslant C \cdot \exp \left(-d^{2}(x, y) / 4\right) \text { for all } x, y \in M
$$

where $d(x, y)$ is the distance function on $M$.

(c) For some point $y_{0} \in M$, there is a constant $K$ such that

$$
\frac{1}{V\left(r, y_{0}\right)} \int_{B\left(r, y_{0}\right)} H_{t}\left(x, y_{0}\right)<K \cdot H_{t}\left(y_{0}, y_{0}\right) \text {. }
$$

Then, if $M$ has subexponential growth, 0 is in the spectrum of $\Delta$.

Received by the editors March 2, 1980 and, in revised form, August 25, 1980 and October 20, 1980. AMS (MOS) subject classifications (1970). Primary 58G25.

${ }^{1}$ Partially supported by NSF grant MCS7802679. 
Condition (b) is a special case $(T=1)$ of an inequality that was studied by Donnelly in [4], where it was verified for a large class of manifolds. We remark that his proof gives condition (b) as a consequence of bounds on the local geometry of $M$. More precisely, if there is a positive number $r_{0}$ such that the injectivity radius at each point is greater than $r_{0}$, then one constructs the parametrix $H_{l}(x, y), l>n / 2$, in terms of the local geometry of $M$. If one then has the estimate

$$
\left|\left(\Delta_{x}+\partial / \partial t\right) H_{l}(x, y, t)\right|<(\text { const }) t^{l-n / 2}
$$

for $0<t<1$, where (const) is independent of $x, y, t$, then the argument of [4] establishes (b).

Unfortunately, we do not know whether (c) is similarly a consequence of bounds on the local geometry of $M$, for instance, we do not know whether (a) and (b) imply (c). It is established in [2] that if $M$ is a "model space", for instance a rank 1 symmetric space, then $H_{t}\left(x, y_{0}\right)$ is a decreasing function of the distance from $x$ to $y_{0}$, so that (c) follows with $K=1$.

We prove the Theorem in $\$ 1$. In $\$ 2$ we present some remarks concerning the situation where we allow $M$ to have positive exponential growth.

We remark that the Theorem is the analogue in Riemannian geometry of the following group-theoretic result: If $G$ is a finitely-generated group with subexponential growth, then $G$ is amenable. The best proof of this, together with definitions of the terms involved, may be found in [6. The relationship between the group theory and the Riemannian geometry may be found in [1] and [8].

A result similar to the Theorem has been proved by Cheng and Yau [3] under the more restrictive assumption that $M$ has polynomial growth, but without assumptions such as (a)-(c) above. Their technique is to select test functions constructed from the distance function on $M$, and is more elementary than that presented here.

For other results using the heat kernel on noncompact manifolds, see [2], [4], [5].

The author would like to thank the referee for many valuable remarks, including bringing to the author's attention some of the references cited above, and acknowledges with pleasure helpful conversations with Jeff Cheeger and Scott Wolpert.

1. Proof of the Theorem. The following properties of the heat kernel are well known. For proofs, the reader is referred to [2] and [4].

Proposition 1. (1)

$$
\int_{M} H_{t}\left(x, y_{0}\right) d x=1 \text { for all } y_{0} \in M, t>0 .
$$

$$
H_{t}(x, y)=H_{t}(y, x)>0 \text { for all } x, y \in M, t>0 .
$$

(3) (Convolution Law)

$$
H_{s+t}(x, y)=\int_{M} H_{s}(x, z) \cdot H_{t}(z, y) d z, \quad s, t>0 .
$$

Now, for $0<\alpha<1$, let $r_{\alpha}(t, y)$ be the function of $t$ and $y$ defined as follows: If $B(r, y)$ is the ball of radius $r$ about $y$, we have

$$
\int_{B\left(r_{\alpha}(t, y), y\right)} H_{t}(x, y) d x=\alpha^{t} .
$$


LEMMA 1. If $M$ satisfies conditions (a) and (b), then for all $\alpha$ there is a $k_{\alpha}$ such that $r_{\alpha}(1, y)<k_{\alpha}$ for all $y \in M$.

Proof. From the lower bound given in (a) for the sectional curvature, it is a standard result in comparison theory (see [4] or [9]) that there are constants $C$ and $\mu$ such that

$$
V(r, y)<C \cdot e^{\mu r} \text { for all } y \in M .
$$

We now apply condition (b) to evaluate

$$
\begin{aligned}
\int_{M-B\left(r_{0} y\right)} H_{1}(x, y) d x & <\int_{M-B\left(r_{0} y\right)} C \exp \left(\frac{-d^{2}(x, y)}{4}\right) d x \\
& <\int_{r_{0}}^{\infty} C \exp \left(\frac{-r^{2}}{4}\right) V(r, y) d r \\
& <\text { (const) } \int_{r_{0}}^{\infty} \exp \left(\frac{-r^{2}}{4}+\mu \cdot r\right) d r
\end{aligned}
$$

where the second inequality comes from replacing the shell $r<d(x, y)<r+\varepsilon$ with the ball $B(r+\varepsilon, y)$, which has strictly greater volume.

Now the right-hand side is clearly integrable, so that taking $r_{0}$ sufficiently large, we have

$$
\int_{M-B\left(r_{0 y} y\right)} H_{1}(x, y) d x<1-\alpha .
$$

Applying (i) of Proposition 1, this gives

$$
\int_{B\left(r_{0} y\right)} H_{1}(x, y) d x>\alpha,
$$

which says that $r(1, y)<r_{0}$, establishing the lemma.

LEMMA 2. Under the hypotheses of Lemma 1, for all positive integers $n$, we have

$$
r_{\alpha}(n, y) \leqslant n \cdot k_{\alpha} \text { for all } y \in M .
$$

Proof. This follows from the Convolution Law. Indeed, we have

$$
\begin{aligned}
& \int_{B\left(n \cdot k_{\alpha} y\right)} H_{n}(x, y) d x \\
&= {\left[\int_{M \times \cdots \times M \times B\left(n k_{\alpha}, y\right)} H_{1}\left(y, x_{1}\right) \cdot H_{1}\left(x_{1}, x_{2}\right) \cdot \ldots \cdot H_{1}\left(x_{n-1}, x_{n}\right) d x_{1} \ldots d x_{n}\right] } \\
& \geqslant {\left[\int _ { B ( k _ { \alpha } , y ) } H _ { 1 } ( y , x _ { 1 } ) \cdot \left[\int_{B\left(k_{\alpha}, x_{1}\right)} H_{1}\left(x_{1}, x_{2}\right)\right.\right.} \\
&\left.\left.\cdot\left[\ldots \int_{B\left(k_{\alpha}, x_{n-1}\right)} H_{1}\left(x_{n-1}, x_{n}\right)\right] \ldots\right] d x_{1} \ldots d x_{n}\right] .
\end{aligned}
$$

where the first equality comes from (2) and (3) of Proposition 1, and the inequality comes from the positivity of $H_{1}(x, y)((3)$ of Proposition 1), and the obvious fact that $B\left(k_{\alpha}, x_{n-1}\right) \subseteq B\left(n \cdot k_{\alpha}, y\right)$. 
According to Lemma 1 , each term in the iterated integral is $>\alpha$, so the entire expression is $\geqslant \alpha^{n}$.

Thus $r_{\alpha}(n, y) \leqslant n \cdot k_{\alpha}$ for all $n$, and the lemma is established.

Proof of TheOREM. Suppose that the spectrum of $\Delta$ is bounded below by $\lambda>0$, and let $y_{0}$ be any point in $M$.

By the spectral representation of $H_{t}(x, y)$ (see [2]), one sees easily that

$$
H_{t}\left(y_{0}, y_{0}\right)<\left(H_{1}\left(y_{0}, y_{0}\right)\right) \cdot e^{-\lambda(t-1)} \text { for } t>1 \text {. }
$$

Applying condition (c), we have

$$
\frac{1}{V\left(r, y_{0}\right)} \int_{B\left(r, y_{0}\right)} H_{t}\left(x, y_{0}\right) d x<H_{t}\left(y_{0}, y_{0}\right)<\left(H_{1}\left(y_{0}, y_{0}\right)\right) e^{-\lambda(t-1)} .
$$

We now substitute $r=r_{\alpha}\left(t, y_{0}\right)$ to get

$$
\frac{1}{V\left(r_{\alpha}\left(k, y_{0}\right), y_{0}\right)} \cdot \alpha^{t}<\left(H_{1}\left(y_{0}, y_{0}\right)\right) e^{-\lambda(t-1)},
$$

or, equivalently,

$$
\begin{aligned}
e^{(\lambda+\log (\alpha))} t & \leqslant H_{1}\left(y_{0}, y_{0}\right) \cdot V\left(r_{\alpha}\left(k, y_{0}\right), y_{0}\right) e^{\lambda} \\
& \leqslant(\text { const }) e^{\mu \cdot r_{\alpha}\left(t, y_{0}\right)}
\end{aligned}
$$

where $\mu$ is greater than the exponential growth of $M$, and (const) depends on $\mu$.

Choosing $\alpha$ sufficiently close to 1 so that $\lambda+\log (\alpha)>0$, we now apply Lemma 2 to get

$$
e^{[\lambda+\log (\alpha)] \cdot n}<\text { (const) } e^{\mu \cdot k_{\alpha} \cdot n}
$$

for $n$ a positive integer. Choosing $\mu<(\lambda+\log (\alpha)) / k_{\alpha}$, which we may do since $M$ has subexponential growth, gives a contradiction for $n$ sufficiently large. This contradiction establishes the Theorem.

2. Some remarks. It follows from the proof that if $M$ satisfies conditions (a)-(c) above, and if $\lambda$ denotes the greatest lower bound of the spectrum of $\Delta$ and $\mu>0$ the exponential growth of $M$, then we have

$$
\lim _{t \rightarrow \infty} t^{-1} r_{\alpha}\left(t, y_{0}\right)>(\lambda+\log (\alpha)) / \mu \text {. }
$$

This suggests that the ratio $\lambda / \mu$ is an interesting invariant of the heat flow of $M$; roughly speaking, $\lambda / \mu$ estimates the linear growth of the radius of a ball containing some fixed amount of heat.

One checks easily that the ratio $\lambda / \mu^{2}$ is unchanged by multiplying the metric by a constant: multiplying the metric by a constant $k$ multiplies $\Delta$, and hence $\lambda$, by $1 / k^{2}$; and multiplies the volume of a metric ball $B(r)$ by $k^{n}$, while multiplying the radius of the ball by $1 / k$. The net effect is to multiply $\mu$ by $1 / k$, leaving the ratio $\lambda / \mu^{2}$ unchanged.

More generally, one may ask whether $\lambda / \mu^{2}$ remains unchanged under more complicated perturbations of the metric. In particular, if $M$ is the universal cover of a compact manifold $N$, is $\lambda / \mu^{2}$ independent of the metric chosen on $N$ ?

One may compute $\lambda / \mu^{2}$ readily in a few cases. According to McKean [7], (see also [10]), if $M$ is a simply-connected space of dimension $n$, all of whose sectional 
curvatures are $<-\kappa<0$, then we have the estimate $\lambda>(n-1)^{2} \kappa / 4$; we have equality if $M$ has constant negative curvature.

According to [8] or [9], for spaces of constant negative curvature $-\kappa$, we have $\mu=(n-1) \kappa^{1 / 2}$.

Thus $\lambda / \mu^{2}=1 / 4$ for hyperbolic space of dimension $n$.

\section{REFERENCES}

1. R. Brooks, The fundamental group and the spectrum of the Laplacian (to appear).

2. J. Cheeger and S. T. Yau, A lower bound for the heat kernel (preprint).

3. S. Y. Cheng and S. T. Yau, Differential equations on Riemannian manifolds and their geometric applications, Comm. Pure Appl. Math. 28 (1975), 333-354.

4. H. Donnelly, Asymptotic expansions for the compact quotients of properly discontinuous group actions, Illinois J. Math. 23 (1979), 485-496.

5. Stability theorems for the contimuous spectrum of a negatively curved manifold (preprint).

6. F. P. Greenleaf, Invariant means on topological groups and their applications, Van Nostrand Reinhold, New York, 1969.

7. H. P. McKean, An upper bound to the spectrum of $\Delta$ on a manifold of negative curvature, J. Differential Geom. 4 (1970), 359-376.

8. J. Milnor, A note on curvature and the fundamental group, J. Differential Geom. 2 (1968), 1-7.

9. R. Bishop and R. J. Crittenden, Geometry of manifolds, Academic Press, New York, 1964.

10. S. T. Yau, Isoperimetric constants and the first eigenvalue of a compact Riemannian manifold, Ann. Sci. Ecole Norm. Sup. 8 (1975), 487-507.

Department of Mathematics, University of Maryland, College Park, Maryland 20742 